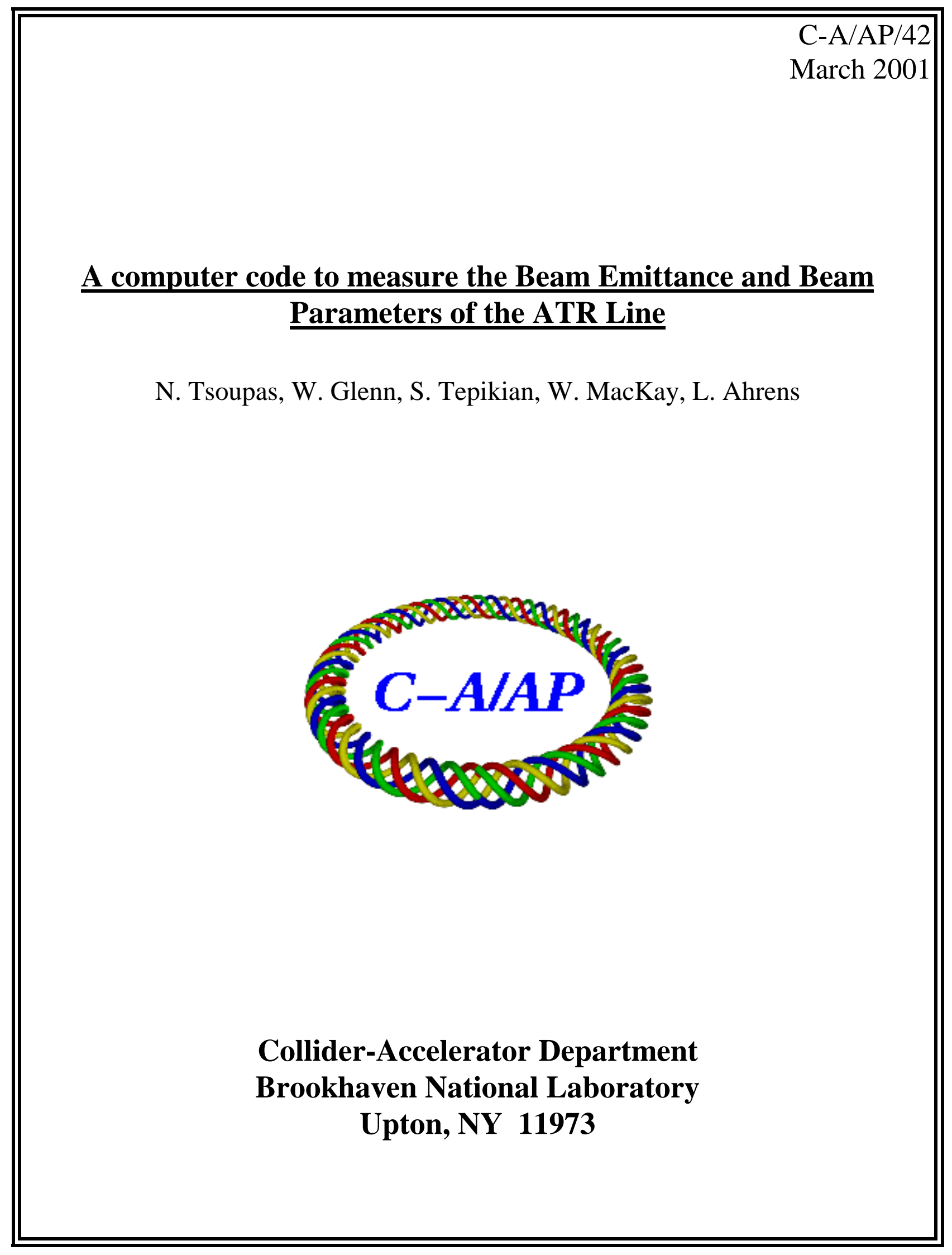




\title{
A computer code to measure the Beam Emittance and Beam Parameters of the ATR Line
}

\author{
N. Tsoupas, W. Glenn, S. Tepikian, W. Mackay, L. Ahrens
}

\begin{abstract}
During beam injection from the Alternating Gradient Synchrotron (AGS) to the Relativistic Heavy Ion Collider (RHIC), the beam parameters $\left(\beta_{\mathrm{x}, \mathrm{y}}, \alpha_{\mathrm{x}, \mathrm{y}}\right)$ of the AGS to RHIC (ATR) transfer line $[1,2,3,4]$ at the RHIC injection point ${ }^{1}$, should "match" those of the RHIC synchrotron at the same point.

A good beam "matching" requires the knowledge of the optics of the ATR transfer line, which depends partly on the beam parameters $\left(\beta_{x, y}, \alpha_{x, y}\right)$ at the origin of the ATR transfer line.

These beam parameters were originally determined with theoretical calculations [5] (before the installation of the ATR line), and presently, (after the completion of the ATR line) can be determined experimentally.

This note addresses the following tasks:

a) Outlines the theory of a method which is currently used to obtain the beam parameters $\left(\beta_{x, y}, \alpha_{x, y}\right)$ and the transverse beam emittance $\left(\varepsilon_{x, y}\right)$ at any given point along the ATR line.

b) Provides a description of the computer code which uses an algorithm based on the theory described in (a) above, to calculate these beam parameters.

c) Presents values of the emittance and beam parameters that have been calculated by using measured beam profiles as input data in the computer code mentioned in (b) above, and compares the beam parameters with theoretically calculated ones.

d) Provides suggestions on future improvements of the computer code and on data collection methods which will enable the experimental determination of the dispersion functions $\left(\eta_{x, y}, \eta_{x, y}^{\prime}\right)$.

\section{Theoretical Formalism of the Method for the Measurement of Beam Emittance and Beam parameters in a Beam Transfer Line}

The method which is applied to measure the beam parameters/emittance is based upon two main assumptions.

First the beam transport is accomplished with zeroth order (drift space, and dipole) and first order (quadrupole) magnetic elements, and second, the particle distribution within the beam is "normal" (Gaussian) in any of its phase-space coordinates.

The first assumption is expressed by the relation

$$
\mathbf{r}_{\text {out }}=\mathbf{R}_{\mathbf{r}_{\text {in }}}
$$

1 The RHIC injection point is defined here along the RHIC ideal closed orbit and at distance $0.197 \mathrm{~m}$ from the exit of the Lambertson type injection septum magnet. MAD8_lat_blue-atr.madout 
The symbols $\mathrm{R}$ in eq. 1 represents the $6 \mathrm{x} 6$ linear matrix which transforms the phase space coordinates $\mathbf{r}_{\mathrm{in}}=\left(\mathrm{x}, \mathrm{x}^{\prime}, \mathrm{y}, \mathrm{y}^{\prime}, \delta l / l, \delta p / p\right)$ of a single particle at the entrance of a magnetic element or beam line to those coordinate $\mathbf{r}_{\mathrm{out}}=\left(\mathrm{x}, \mathrm{x}^{\prime}, \mathrm{y}^{\prime}, \mathrm{y}^{\prime}, \delta l / l, \delta p / p\right)$ at the exit of the beam line, under a linear transformation.

Under the second assumption the beam distribution at any point along the transport line, can be described mathematically by a six dimensional beam ellipsoid which can be expressed by a 6x6 symmetric matrix (the $\sigma$-matrix). This beam ellipsoid transforms between two points along the beam line by the transformation shown in the relation (2).

$$
\sigma_{\text {out }}=\mathrm{R} \sigma_{\text {in }} \mathrm{R}^{\mathrm{T}}
$$

In equation 2 above, $\sigma_{\text {in }}$ is a $6 \times 6$ matrix which represents the six dimensional beam ellipsoid [6] at the entrance of a linear magnetic element or a beam line which consists of linear elements, and $\sigma_{\text {out }}$ the beam ellipsoid at the exit of the magnetic element or the beam line.

It is the property of a "normal" distribution to transform into another "normal" distribution under a linear transformation shown by the relation (2).

The symbol $\mathrm{R}$ is the linear transport matrix ( $\mathrm{R}$-matrix) and depends on the location, the geometry, and the strength of the magnetic elements of the beam line. The symbol $\mathrm{R}^{\mathrm{T}}$ in the relation (2) represents the transpose matrix of $\mathbf{R}$.

A complete description of the beam ellipsoid represented by the $\sigma$-matrix requires the knowledge of 36 elements. The symmetry $\left(\sigma_{i j}=\sigma_{j i}\right)$ of the $\sigma$-matrix reduces the number of elements to 21 and the following assumptions ( 1 to 3 below) reduce the number of the unknown elements to 9 .

1) The initial beam ellipsoid is uncoupled (the $x, x$ ' coordinates are not correlated with any of the $y, y^{\prime}$ coordinates).

2) The beam transport line is free of linear coupling (both the dipoles and the quadrupoles maintain midplane symmetry thus introducing no skew quadrupole component).

3) No beam acceleration is taking place along the beam line which consist of magnetic elements only.

These assumptions reduce the $\sigma$-matrix of the beam ellipsoid, and the R-matrix into the matrices shown in the equations (3) an (4) below which show explicitly the non-zero matrix elements.

$$
(\sigma)=\begin{array}{cccccc}
\sigma_{11} & \sigma_{12} & 0 & 0 & \sigma_{15} & \sigma_{16} \\
\sigma_{12} & \sigma_{22} & 0 & 0 & \sigma_{25} & \sigma_{26} \\
0 & 0 & \sigma_{33} & \sigma_{34} & \sigma_{35} & \sigma_{36} \\
0 & 0 & \sigma_{34} & \sigma_{44} & \sigma_{45} & \sigma_{46} \\
\sigma_{15} & \sigma_{25} & \sigma_{35} & \sigma_{45} & \sigma_{55} & \sigma_{56} \\
\sigma_{16} & \sigma_{26} & \sigma_{36} & \sigma_{46} & \sigma_{56} & \sigma_{66}
\end{array}
$$




$$
(\mathrm{R})=\begin{array}{cccccc}
R_{11} & R_{12} & 0 & 0 & 0 & R_{16} \\
R_{12} & R_{22} & 0 & 0 & 0 & R_{26} \\
0 & 0 & R_{33} & R_{34} & 0 & R_{36} \\
0 & 0 & R_{43} & R_{44} & 0 & R_{46} \\
R_{51} & R_{52} & R_{53} & R_{54} & 1 & R_{56} \\
0 & 0 & 0 & 0 & 0 & 1
\end{array}
$$

The correspondence between the subscripts appearing in equations 3 and 4 and the physical quantities they represent is given by the relation (5) below

$$
(1,2,3,4,5,6) \Leftrightarrow>\left(\mathrm{x}, \mathrm{x}^{\prime}, \mathrm{y}, \mathrm{y}^{\prime}, \delta / / l, \delta p / p\right)
$$

The physical meaning of the elements of the $\sigma$-matrix is given below:

$\left(\sigma_{11}\right)^{1 / 2}=(\mathrm{x})_{\mathrm{sd}} \quad$ (the standard deviation of the particle distribution in $\left.\mathrm{x}\right)$

$\left(\sigma_{22}\right)^{1 / 2}=\left(\mathrm{x}^{\prime}\right)_{\mathrm{sd}} \quad$ (the standard deviation of the particle distribution in $\left.\mathrm{x}^{\prime}\right)$

$\left(\sigma_{66}\right)^{1 / 2}=(\delta p / p)_{\text {sd }}$ (the standard deviation of the particle distribution in $\left.\delta p / p\right)$

$\left(\sigma_{12}\right)$ is related to the correlation coefficient $\left(\mathrm{r}_{12}\right)$ of $\left(\mathrm{x}, \mathrm{x}^{\prime}\right)$ coordinates $\left(\mathrm{r}_{12}\right)=\left(\sigma_{12}\right) /\left(\sigma_{11} \sigma_{22}\right)^{1 / 2}$ and providesinformation on the orientation of the ellipse which is the projection of the coordinates of the beam ellipsoid on the $\mathrm{x}, \mathrm{x}$ ' plane.

$\left(\sigma_{16}\right)$ is related to the correlation coefficient $\left(\mathrm{r}_{16}\right)$ of $(\mathrm{x}, \delta p / p)$ coordinates $\left(\mathrm{r}_{16}\right)=\left(\sigma_{16}\right) /\left(\sigma_{11} \sigma_{66}\right)^{1 / 2}$ and provides information on the orientation of the ellipse which is the projection of the ellipsoid in the $\mathrm{x}, \delta p / p$ plane.

The relation $\sigma_{16}=\eta_{\mathrm{x}} \sigma_{66}$ is also valid (APPENDIX 1) where $\eta_{\mathrm{x}}$ is the dispersion function.

$\left(\sigma_{26}\right)$ is related to the correlation coefficient $\mathrm{r}_{26}$ of $\left(\mathrm{x}^{\prime}, \delta p / p\right)$ coordinates $\left(\mathrm{r}_{26}\right)=\left(\sigma_{26}\right) /\left(\sigma_{22} \sigma_{66}\right)^{1 / 2}$ and provides information on the orientation of the ellipse which is the projection of the ellipsoid in the $\mathrm{x}^{\prime}, \delta p / p$ plane.

The relation $\sigma_{26}=\eta_{x}^{\prime} \sigma_{66}$ is also valid (APPENDIX 1) where $\eta_{x}^{\prime}$ is the angular dispersion function.

Similar interpretation holds for the rest of the elements of the $\sigma$-matrix.

The R-matrix elements which appear in equations (1) and (2), are the first order coefficients of the Taylor series expansion of the output coordinates in terms of the input coordinates.

For example:

$$
x_{i, \text { out }}=\left(\partial x_{i, \text { out }} / \partial x_{j, \text { in }}\right) x_{j, \text { in }}=R_{i, j} x_{j, \text { in }}
$$


In the equation (6) above, the notation shown in (7) is assumed and the summation over repeated indices is implied.

$$
\left(\mathrm{x}_{1}, \mathrm{X}_{2}, \mathrm{x}_{3}, \mathrm{x}_{4}, \mathrm{x}_{6}\right) \Leftrightarrow\left(\mathrm{x}, \mathrm{x}^{\prime}, \mathrm{y}, \mathrm{y}^{\prime}, \delta p / p\right)
$$

The assumptions made earlier, greatly simplify the expression of the beam ellipsoid in terms of the $\sigma$-matrix as well as the transformation of the beam ellipsoid by equation (2).

For example the general form of the $\left(\sigma_{11}\right)_{\text {out }}$ matrix element as derived from equation (2) is given by:

$$
\begin{aligned}
& \left(\sigma_{11}\right)_{\text {out }}=\left(\mathrm{R}_{11}\right)^{2}\left(\sigma_{11}\right)_{\text {in }}+2\left(\mathrm{R}_{11}\right)\left(\mathrm{R}_{12}\right)\left(\sigma_{12}\right)_{\text {in }}+\left(\mathrm{R}_{12}\right)^{2}\left(\sigma_{22}\right)_{\text {in }} \\
& +2\left(\mathrm{R}_{11}\right)\left(\mathrm{R}_{16}\right)\left(\sigma_{16}\right)_{\text {in }}+2\left(\mathrm{R}_{12}\right)\left(\mathrm{R}_{16}\right)\left(\sigma_{26}\right)_{\text {in }}+\left(\mathrm{R}_{16}\right)^{2}\left(\sigma_{66}\right)_{\text {in }} \\
& \left(\sigma_{33}\right)_{\text {out }}=\left(\mathrm{R}_{33}\right)^{2}\left(\sigma_{33}\right)_{\text {in }}+2\left(\mathrm{R}_{33}\right)\left(\mathrm{R}_{34}\right)\left(\sigma_{34}\right)_{\text {in }}+\left(\mathrm{R}_{34}\right)^{2}\left(\sigma_{44}\right)_{\text {in }} \\
& +2\left(\mathrm{R}_{33}\right)\left(\mathrm{R}_{36}\right)\left(\sigma_{36}\right)_{\text {in }}+2\left(\mathrm{R}_{34}\right)\left(\mathrm{R}_{36}\right)\left(\sigma_{46}\right)_{\text {in }}+\left(\mathrm{R}_{36}\right)^{2}\left(\sigma_{66}\right)_{\text {in }}
\end{aligned}
$$

At regions of the beam line where the dispersion and angular dispersion $\left(\eta_{\mathrm{x}, \mathrm{y}}, \eta_{x, y}^{\prime}\right)$ are both equal to zero and the beam ellipsoid is matched to the beam parameters of the beam line (achromatic beam) the expressions in equation ( $8 \mathrm{a}$ and $8 \mathrm{~b}$ ) are further simplified (see APPENDIX 1) to:

$$
\left(\sigma_{11}\right)_{\text {out }}=\left(\mathrm{R}_{11}\right)^{2}\left(\sigma_{11}\right)_{\text {in }}+2\left(\mathrm{R}_{11}\right)\left(\mathrm{R}_{12}\right)\left(\sigma_{12}\right)_{\text {in }}+\left(\mathrm{R}_{12}\right)^{2}\left(\sigma_{22}\right)_{\text {in }}
$$

with the corresponding expression for the vertical plane (y-plane) given by:

$$
\left(\sigma_{33}\right)_{\text {out }}=\left(\mathrm{R}_{33}\right)^{2}\left(\sigma_{33}\right)_{\text {in }}+2\left(\mathrm{R}_{33}\right)\left(\mathrm{R}_{34}\right)\left(\sigma_{34}\right)_{\text {in }}+\left(\mathrm{R}_{34}\right)^{2}\left(\sigma_{44}\right)_{\text {in }}
$$

Equation 9a indicates that at least three independent measurements of the $\left(\sigma_{11}\right)_{\text {out }}$ should be performed in regions of the beam line where the beam is achromatic, in order to determine the following quantities.

$$
\begin{aligned}
& \left(\mathrm{x}_{\mathrm{sd}}\right)_{\text {in }}=\left[\left(\sigma_{11}\right)_{\text {in }}\right]^{1 / 2} \\
& \left(\mathrm{x}_{\text {sd }}\right)_{\text {in }}=\left[\left(\sigma_{22}\right)_{\text {in }}\right]^{1 / 2} \\
& \varepsilon_{\mathrm{x}}=\left[\left(\sigma_{11}\right)_{\text {in }}\left(\sigma_{22}\right)_{\text {in }}-\left(\sigma_{12}\right)_{\text {in }}{ }^{2}\right]^{1 / 2} \\
& \left(\beta_{\mathrm{x}}\right)_{\text {in }}=\left[\left(\sigma_{11}\right)_{\text {in }}\right] /\left[\left(\sigma_{11}\right)_{\text {in }}\left(\sigma_{22}\right)_{\text {in }}-\left(\sigma_{12}\right)_{\text {in }}{ }^{2}\right]^{1 / 2}=\left(\sigma_{11}\right)_{\text {in }} / \varepsilon_{\mathrm{x}} \\
& \left(\alpha_{\mathrm{x}}\right)_{\text {in }}=-\left[\left(\sigma_{12}\right)_{\text {in }}\right] /\left[\left(\sigma_{11}\right)_{\text {in }}\left(\sigma_{22}\right)_{\text {in }}-\left(\sigma_{12}\right)_{\text {in }}{ }^{2}\right]^{1 / 2}=-\left(\sigma_{12}\right)_{\text {in }} / \varepsilon_{\mathrm{x}}
\end{aligned}
$$

The equations (12) to (14) above are used to determine the emittance $\left(\varepsilon_{\mathrm{X}}\right)$ and beam parameters $\left(\beta_{\mathrm{x}}, \alpha_{\mathrm{x}}\right)$ at the entrance of the beam line. 
The emittance $\left(\varepsilon_{\mathrm{x}}\right)$ as expressed by eq. (12) is the area of the (x, $\left.\mathrm{x}^{\prime}\right)$ phase space occupied by $68 \%$ of the particles. This quantity is invariant under the assumptions made earlier (linear transport, no linear coupling).

Similar equations, like equations (10) to (14), are also valid for the corresponding physical quantities of the (y,y')-plane.

\section{Implementation of the Method to calculate beam emittance/parameters}

The determination of the beam emittance $\left(\varepsilon_{\mathrm{x}, \mathrm{y}}\right)$ and the beam parameters $\left(\beta_{\mathrm{x}, \mathrm{y}}, \alpha_{\mathrm{x}, \mathrm{y}}\right)$ requires a minimum of six beam profile measurements (three beam profiles for the horizontal quantities and three for the vertical).

A beam profile, is the projection of the phase space coordinates of the particles within the beam ellipsoid, on the x-axis (horizontal beam profile) or the $y$-axis (vertical beam profile) and is obtained by devices called "flag profile monitors" or "flags".

A "flag" measures the beam distribution on a the x-plane and/or y-plane, which are transverse to the beam direction. The projection of such a distribution on the $\mathrm{x}$ or $\mathrm{y}$ axis provides the required beam profiles from which the standard deviation of the particles distribution can be calculated. A typical horizontal and vertical beam profile which was obtained by a "flag" is shown in fig 1. The beam profiles of the ATR line are obtained with a set of three flags (UF3, UF4, and UF5) which are located along the U-line straight section ${ }^{2}$ which follows the $8^{0}$ bend, and a second set of three flags (WF1, WF2, WF3) which are located along the W-line straight section which follows the $20^{\circ}$ bend.

In both straight sections of the ATR-line, mentioned above, the dispersion functions $\left(\eta_{\mathrm{x}, \mathrm{y}}, \eta_{x}^{\prime}\right)$ are equal to zero, and proper beam matching should yield an achromatic beam. Therefore the relations (9a) and (9b) above are valid.

The following steps are taken to obtain the required beam profiles for the computation of the beam emittance and beam parameters.

1. The beam transport along the ATR line must be acceptable. (Minimum beam losses and the beam trajectory as measured with the BPM's of the ATR line is well centered).

2. The magnet_file containing the present settings of the magnets of the ATR line has been written ("saved") in the directory

“/operations/app_store/Magnet/save/magnet_setting_file_name of your choise".

The saved magnet file "magnet_setting_file_name of your choise" and the path directory leading to the "saved" file, will also resides in the "magnet manager" application. This information of the saved "magnet_setting_file_name of your choise" and the path directory will also be written on a file by the "ATR Flag Profile Monitor" application (see next step) to be used for the calculation of the first order R-matrix elements mentioned earlier.

2 A phase advance of $120^{\circ}$ between the locations of two consecutive flags minimizes the error in the computation of the emittance [7]. 
3. In the "StartUp" menu call the application/start/AGS_Applications/ATR Flag Profile Monitor.

4. Using this application choose a particular flag among the flags mentioned earlier (UF3, UF4,UF5, WF1, WF2, WF3) and "connect" this flag with one of the four available "frame grabers" (fg1,fg2,fg 3, fg 4$)^{3}$.

5. Insert this flag in the beam line and obtain the $\mathrm{x}$ and $\mathrm{y}$ beam profiles.

Observe the fits that the "ATR Flag Profile Monitor" application performs to both $\mathrm{x}$ and $\mathrm{y}$ beam profiles to a Gaussian distribution.

Make sure that the beam profiles do not "saturate the flag with the emitted light" and the beam profile "tails" sit on a "reasonable" background.

To eliminate any saturation and improve the background, adjust the "attenuation_level" and the "black level" respectively. Both adjustments can be made through the "ATR Flag Profile Monitor" application.

Record the optimum settings of the "attenuation_level" and the "black level".

A reasonably good fit of a Gaussian to an experimental beam profile is sown in figure 1.

6. Repeat steps 4 and 5 with a different "flag" connected to the same or a different framegrabber.

7. When steps 5 and 6 are completed, turn on "emit" in the "ATR Flag Profile Monitor" application window and obtain the beam profiles with a particular flag/frame-grabber combination. The flag application fits Gaussian curves to the beam profiles and computes the standard deviations of the horizontal and vertical profiles.

After the Gaussian fits are performed on the beam profiles, the following information is written on a file:

a) name of the flag (uf1, or uf 2 etc....)

b) horizontal and vertical standard deviations of the beam profiles

c) the magnet file and the path to the magnet file (obtained from the magnet manager; see step 2).

The inclusion of the "magnet file name" with each beam profile measurement is necessary, because the beam profiles may be taken with different magnet settings. In this case the "magnet file name" should also be saved using the "magnet manager" application (see step 2) prior to each profile measurements.

The above information a) b), c) is written in a single line in a file which has a "timestamp" for file name; for example, "Tue_Apr-04-2000_17-20-33".

This file resides in the directory /operations/app_store/FPM/fpmChart/Emit/

Repeat steps 4 to 6 to obtain additional profiles with the same flag. When a good set of profiles is obtained turn off "emit" in the flag application.

8. Retract the flag used in step 7 above and repeat step 7 with a different flag/frame-grabber which was selected in step 4 and 5 combination.

Remember to apply the optimum settings of the "attenuation_level" and the "black level" as determined in step 5 for this particular flag/frame-grabber combination.

9. Repeat step 8 until all required beam profiles are obtained.

3 At the present time there are four frame grabbers available. 


\section{emit_ATR: A program to calculate Beam Emittance and Parameters in the ATR Transfer Line. (Description and Operation of the Program)}

This program is written in a $\mathrm{C}++$ computer language and calculates the beam emittance and beam parameters from beam profiles collected at various locations of the ATR transfer line. The algorithm used by the program is based on the formalism described in a previous section. The flow-chart diagram of the computer program is shown in figure 2 and is discussed below.

1. Upon the execution of the program "emit_ATR" the program asks the user for the file name of the file which contains the profiles that were collected using the flag profile application mentioned earlier.

2. The code asks the user for the location along the ATR line where the emittance and the beam parameters should be calculated.

This location should exist under a particular name (for example "begin" for the location at the beginning of the ATR line, "uf1" for the location of the flag UF1 etc) in the transport code which is used to calculate the matrix elements Rij which are used in equations (9a) and (9b) above.

3. The code calculates the first order matrix elements $R_{i j}$ between the location provided in step 2 above, and the particular flag. These matrix elements are based on the magnet file which is associated with the optics of the ATR line at the instant the profiles were taken (see step 2 in previous section), and are calculated by the computer code "bl" [8] of the ATR line or the MAD computer code [9] which are both set to describe the first order optics of the ATR line.

The user selects "bl" or "MAD", to be used in the calculations of the first order matrix elements.

4. The code displays on the terminal and prints on a file "emit.res" the emittance $\left(\varepsilon_{\mathrm{x}}, \varepsilon_{\mathrm{y}}\right)$, and the beam parameters at the "start" location chosen in step 2 and at the location of the flags which were used in the emittance calculations.

\section{Some Results from the beam emittance/parameters measurement}

Emittance measurements using the ATR transfer line were performed during the commissioning of the ATR line [7].

Some recent results from the beam emittance/parameters measurements which were performed during the RHIC 2000 run, are shown in Table 1.

The beam parameters shown in Table 1 (columns 2 to 5) correspond to those at the beginning of the ATR-line.

The first column in TABLE 1 shows the measurement \# and the flags which were used in the measurements. In particular the results of the first row labeled "1 u\&w-flags" correspond to the measurements performed with the flags uf3, uf4, uf5, of the U-line, and wf1, wf2, wf3 of the Wline.

Both sets of flags $(u$ and $w)$ are in regions where the dispersion functions $\left(\eta_{\mathrm{x}, \mathrm{y}}, \eta_{x}^{\prime}\right)$ are assumed to be zero. 
The results of the second and third lines in TABLE 1 correspond to flag measurements of the Uline only, and $\mathrm{W}$-line only, respectively. The data in each of the rows $(2,3,4)$, were taken on different dates.

The last row of the Table 1 shows the theoretically calculated beam parameters [5], at the same location (beginning of the ATR-line).

Table 1

\begin{tabular}{|cc|c|c|c|c|c|c|}
\hline & Meas \# & $\beta_{\mathrm{x}}[\mathrm{m}]$ & $\alpha_{\mathrm{x}}$ & $\beta_{\mathrm{y}}[\mathrm{m}]$ & $\alpha_{\mathrm{y}}$ & $\varepsilon_{\mathrm{x}}[\pi \cdot \mathrm{m} . \mathrm{rad}]$ & $\varepsilon_{\mathrm{y}}[\pi \cdot \mathrm{m} . \mathrm{rad}]$ \\
\hline 1 & u\&w-flags & 36.7 & -4.5 & 7.7 & 1.2 & 7.3 & 7.6 \\
\hline 2 & u-flags & 28.6 & -3.3 & 10.5 & 1.5 & 8.9 & 7.1 \\
\hline 3 & w-flags & 28.5 & -3.4 & 9.2 & 0.5 & 9.8 & 9.6 \\
\hline \multicolumn{2}{|c|}{ Calculations } & 37.5 & -4.1 & 6.5 & 0.85 & & \\
\hline
\end{tabular}

Table 1: The beam parameters at the beginning of the ATR line. The results from the rows 2, 3 and 4 are obtained from calculations based on beam profile measurements. The results from the row 5 correspond to the calculations based on theory (see text).

The beam parameters at the RHIC Injection Point (corresponding to the measurements of Table 1) are shown in Table 2. These results in Table 2 were obtained from the corresponding results of Table 1, when the beam parameters are transported, from the beginning of the ATR line to the RHIC Injection Point, by means of the ATR Transport Matrix.

Table 2

\begin{tabular}{|cc|c|c|c|c|}
\hline & Meas \# & $\beta_{\mathrm{x}}[\mathrm{m}]$ & $\alpha_{\mathrm{x}}$ & $\beta_{\mathrm{y}}[\mathrm{m}]$ & $\alpha_{\mathrm{y}}$ \\
\hline 1 & u\&w-flags & 26.3 & -1.5 & 15.5 & 0.66 \\
\hline 2 & u-flags & 27.3 & -1.2 & 11.3 & 0.39 \\
\hline 3 & w-flags & 24.8 & -1.2 & 14.2 & 1.4 \\
\hline \multicolumn{2}{|c|}{ Calculations } & 37.7 & -1.75 & 16.5 & 0.95 \\
\hline
\end{tabular}

Table 2: The beam parameters at the beginning of the ATR line. The results from the rows 2, 3 and 4 are obtained from calculations based on measurements. The results from row 5 correspond to the calculations based on theory (see text).

The error corresponding to the value of $\beta_{\mathrm{x}, \mathrm{y}}$ is $\sim 20 \%$.

In order to calculate the error, many profile measurements for each of the flags were obtained. From these measurements an average beam profile $\mathrm{x}_{\text {ave }}$ and a standard deviation $\mathrm{x}_{\mathrm{sd}}$ was calculated for each flag, and the error in calculating a particular quantity, $\delta \beta$ in this case, was calculated using the relation (15) below.

$$
\delta \beta_{x, y}=\sum \frac{\partial \beta_{x, y}}{\partial x_{i}}\left(\delta x_{i}\right)_{s d}
$$

Each of the terms $\left(\partial \beta_{\mathrm{x}, \mathrm{y}} / \partial \mathrm{x}_{\mathrm{i}}\right) \delta\left(\mathrm{x}_{\mathrm{i}}\right)_{\mathrm{sd}}$ in equation (15) above corresponds to the location of a flag and was calculated using the maximum absolute value of the difference $\left\{\beta\left(x_{a v e}\right)-\beta\left(x_{a v e}-x_{s d}\right)\right\}$. This error includes the error due to the fluctuations of the power supplies current which powers the magnets of the ATR line. 
The errors on the quantities $\delta \alpha_{x, y}$ and $\delta \varepsilon_{x, y}$ can be calculated in the same way.

We also assumed that the ATR magnets were positioned without any errors along the ATR line. Any errors due to magnet location must be treated as systematic errors.

An improved method to calculate the errors in beam parameters/emittance has to be implemented (see following section).

\section{Future work and Improvements}

- To incorporate in the emitt_ATR program a routine which computes the error in calculating the beam parameters and emittance from the error in measuring beam profiles

The calculation of the horizontal $\left(\varepsilon_{x}, \beta_{x}, \alpha_{x}\right)$, or vertical $\left(\varepsilon_{y}, \beta_{y}, \alpha_{y}\right)$, emittance and beam parameters requires a minimum of three beam profiles which must be obtained at different locations along the ATR line or at the same location but with different beam optics (different quad settings) between the "start" location and the "flag". An error in the beam parameters/emittance based on only three beam profiles is not reliable.

One way to assign an error in the measured quantities, is to measure a set of more than three beam profiles. The "emit-ATR" program can currently use a large number ( 70) of beam profiles to compute the required beam emittance and parameters by the method of least squares. The "chi square" obtained from the solution of n-equations with 3 unknowns can be used in the calculation of the errors of the various physical quantities to be determined.

- The program "emit_ATR" to be incorporated in the "ATR_Flag Profile Monitor" application

The program "emit_ATR" is currently running independently of the "ATR_Flag Profile Monitor" application which provides the required beam profiles. It is however desired to be integrated into the "ATR_Flag Profile Monitor" application.

- Implement a faster method to measure the beam parameters/beam profiles

The simultaneous insertion of three or four flags (currently there are four frame-grabbers) can provide all the required beam profiles by using a single bunch in the ATR-line.

In a "single-bunch multiple-flag measurement" the multipole scattering of the beam in the upstream flags will affect the beam profile at the downstream flags. The effect of the multiple scattering can be taken into account although at "high beam energies" (RHIC injection energies), this effect is well within the error in calculating the beam emittance .

- Modify the "emit_ATR" program to enable the determination of the dispersion functions $\left.\left(\eta_{x}\right)_{\text {in }},\left(\eta^{\prime}\right)^{\prime}\right)_{\text {in }}$

The calculations for the beam emittance are based on the equations (9a) and (9b) which assume that the beam is achromatic at the location of the flags.

In the more general case however, the eq. (8a) and (8b) may be used. In these equation the assumption that the beam is achromatic at the location of the flags is not valid, and the beam may be either chromatic or achromatic.

The solution of these more general equations may provide values for the quantities $\left(\sigma_{16}\right)_{\text {in }}$ $\left(\sigma_{26}\right)_{\text {in }}$, and $\left(\sigma_{66}\right)_{\text {in, }}$, which in turn can be used to calculate the values and the beam 
momentum spread $\delta p / p\left\{\left(\sigma_{66}\right)_{\text {in }}=(\delta p / p)^{2}\right\}$ and the dispersion functions $\left(\eta_{\mathrm{x}}\right)_{\text {in }}$ and, $\left(\eta^{\prime}{ }_{\mathrm{x}}\right)_{\text {in }}$, at the entrance of the beam line $\left\{\left(\sigma_{16}\right)_{\text {in }}=\left(\eta_{\mathrm{x}}\right)_{\text {in }}\left(\sigma_{66}\right)_{\text {in }}\right.$ and $\left.\sigma_{26}=\left(\eta^{\prime}{ }_{x}\right)_{\text {in }}\left(\sigma_{66}\right)_{\text {in }}\right\}$.

The profile data from the flags uf 3 , uf4, uf5, wf1, wf2, wf3 corresponding to the second row of TABLE 1 indeed provide six equations to calculate the additional three unknowns. The solution of these equations however could not provide results with physical meaning. Part of the reason may be the fact that the dispersion function at the locations of the flags is almost zero, therefore the contribution of the dispersion to the beam profiles is smaller then the beam-profile measurement error.

A way to alter the situation, is to introduce significant dispersion at the location of the flags.

The above statements are also true for the vertical plane which, in the ATR line, lacks of large vertical bends, therefore the beam in this plane is rather achromatic.

\section{- Employ additional flags of the ATR line for beam profile measurements}

In addition to the "flags" (UF3, UF4, UF5) of the U-line and the "flags (WF1, WF2, WF3) of the W-line, there are two more "flags" (UF1, and UF2) located in the upstream section of the U-line, and four more flags (XF1,XF2, YF1, and YF2) located in the X-line and Y-line respectively, which are also available and can be used for measurements of the beam profiles. In this case where the measurements of the beam profiles are performed in regions where the beam is achromatic (flags UF1, UF2, XF1,XF2) the equations (8a) and (8b) are to be used for the calculation of the beam parameters.

- Visual presentation of the measured and calculated beam ellipses at the "start" location, and of the location of the flags

\section{Alternative way of obtaining beam emittance}

An alternative method of calculating beam emittance has also been used in the ATR-line. The method is based (see fig. 3) in varying the quadrupole strength (in this particular case the UQ13 of the U-line) and measuring the beam profile (horizontal in this case) as a function of the Quadrupole strength.

The relation between the emittance and the beam profiles at the location of the flags uf4 and uf5 (when a beam waist is formed at uf5) is given by:

$$
\varepsilon_{\mathrm{x}}=\left\{\left[\sigma_{11}\left(\text { at uf4) }-\sigma_{11}(\text { at uf } 5, \text { waist })\right] \cdot \sigma_{11}(\text { at uf5,waist })\right\}^{1 / 2} / \mathrm{s}\right.
$$

In equation 16 the symbol $\sigma_{11}$ is the square of the standard deviation of the Gaussian beam profile and $\mathrm{s}$ is the distance between the two flags. It is assumed that the distance between the location of the flag uf4 and the center of the quadrupole UQ13 is small compared to the distance $s$.

The beam profile at the flag uf5 as function of the quadrupole UQ13 strength is shown in figure 4 . The minimum value of the parabolic curve which fits the experimental profiles, is the beam waist, used in eq. 16. 


\section{ACKNOWLEDGMENTS}

We thank Dr. Steven Peggs for suggesting the write-up and implementation of the "emit-ATR" program. We also like to thank him for taking time to check our results, in calculating the beam parameters and beam emittance, using his own computer code.

\section{REFERENCES}

[1] Focusing and Matching Properties of the AtR Transfer Line N. Tsoupas et. al. BNL, PAC97 Vancouver, BC, May 12-16 (1997)

[2] AGS to RHIC Transfer Line: Design and Commissioning W.W. MacKay et.al. BNL,

European Particle Accelerator Conference 1996 Barcelona Spain (RHIC/AP/103)

[3] Physics of the AGS to RHIC Transfer Line Commissioning L. Ahrens et. al. BNL,

European Particle Accelerator Conference 1996 Barcelona Spain

[4] J. Claus and H. Foelsche "Beam Transfer from AGS to RHIC" RHIC-47, 1988

[5] Closed Orbit Calculations at AGS and Extraction Beam Parameters at H13

N. Tsoupas, H.W. Foelsche, J. Claus and R. Thern, BNL, Oct. 1994, (AD/RHIC/RD-75)

[6] A First and Second Order Matrix Theory for the Design of Beam Transport Systems and Charged Particle Spectometers.

Karl L. Brown SLAC REPORT No. 75

[7] The phase advance between AtR Flags.

S. Peggs, N. Tsoupas “ (RHIC/AP/79 DEC 1995

[8] An Interactive Beam-Line Simulator Module for RHIC

W. W. MacKay Particle Accelerator Conference 12-16 May 1997 p. 2541

[9] Third-Order Transport. A Computer Program for Designing Charged Particle Beam Transport Systems.

D. C. Carey et. al. SLAC-R-95-462 May 1995 


\section{APPENDIX 1}

In this APPENDIX we will prove, for a beam line which is not "linearly coupled" the following two items:

(a) The relations (A1) and (A2) below are valid at any section of the beam line.

$$
\begin{aligned}
& \sigma_{16}=\eta_{x} \sigma_{66} \\
& \sigma_{26}=\eta_{x}^{\prime} \sigma_{66}
\end{aligned}
$$

(b) Equations 8a, and $8 \mathrm{~b}$ are reduced to equations $9 \mathrm{a}$, and $9 \mathrm{~b}$ at any section of a beam line where the beam is achromatic $\left(\eta_{x, y}=0, \eta_{x, y}^{\prime}=0\right.$ and the beam matches the functions of the beam line).

A $\sigma$-matrix at the entrance of a beam line is transformed to a $\sigma$-matrix at the exit by means of the transformation given by eq. (A3)

$$
\sigma_{\text {out }}=\mathrm{R}_{\text {in }} \mathrm{R}^{\mathrm{T}}
$$

The assumption of non-linear coupling reduces the (A3) equation above to two independent equations, one describing the transformation on the x-plane, and the other on the y-plane. The equation which describes the transformation of the beam ellipsoid on the x-plane, is written explicitly by the matrix-equation (A4) below.

$$
\begin{array}{llllllllllll}
\left(\sigma_{11}\right)_{\text {out }} & \left(\sigma_{12}\right)_{\text {out }} & \left(\sigma_{16}\right)_{\text {out }} & R_{11} & R_{12} & R_{16} & \left(\sigma_{11}\right)_{\text {in }} & \left(\sigma_{12}\right)_{\text {in }} & \left(\sigma_{16}\right)_{\text {in }} & R_{11} & R_{21} & 0 \\
\left(\sigma_{12}\right)_{\text {out }} & \left(\sigma_{22}\right)_{\text {out }} & \left(\sigma_{26}\right)_{\text {out }}=R_{21} & R_{22} & R_{26} \bullet\left(\sigma_{12}\right)_{\text {in }} & \left(\sigma_{22}\right)_{\text {in }} & \left(\sigma_{26}\right)_{\text {in }} \bullet R_{12} & R_{22} & 0 \\
\left(\sigma_{16}\right)_{\text {out }} & \left(\sigma_{26}\right)_{\text {out }} & \left(\sigma_{66}\right)_{\text {out }} & 0 & 0 & 1 & \left(\sigma_{16}\right)_{\text {in }} & \left(\sigma_{26}\right)_{\text {in }} & \left(\sigma_{66}\right)_{\text {in }} & R_{16} & R_{26} & 1
\end{array}
$$

The multiplication of the three matrices at the right hand side of equation (A4) yields the following expressions for the four matrix elements which are needed to prove items (a) and (b) above.

$$
\begin{aligned}
& \left(\sigma_{11}\right)_{\text {out }}=R_{11}^{2}\left(\sigma_{11}\right)_{\text {in }}+2 R_{11} R_{12}\left(\sigma_{12}\right)_{\text {in }}+R_{12}^{2}\left(\sigma_{22}\right)_{\text {in }}+2 R_{11} R_{16}\left(\sigma_{16}\right)_{\text {in }}+2 R_{12} R_{16}\left(\sigma_{26}\right)_{\text {in }}+R_{16}^{2}\left(\sigma_{66}\right)_{\text {in }} \\
& \left(\sigma_{16}\right)_{\text {out }}=R_{11}\left(\sigma_{16}\right)_{\text {in }}+R_{12}\left(\sigma_{26}\right)_{\text {in }}+R_{16}\left(\sigma_{66}\right)_{\text {in }} \\
& \left(\sigma_{26}\right)_{\text {out }}=R_{21}\left(\sigma_{16}\right)_{\text {in }}+R_{22}\left(\sigma_{26}\right)_{\text {in }}+R_{26}\left(\sigma_{66}\right)_{\text {in }} \\
& \left(\sigma_{22}\right)_{\text {out }}=R_{21}^{2}\left(\sigma_{11}\right)_{\text {in }}+2 R_{21} R_{22}\left(\sigma_{12}\right)_{\text {in }}+R_{22}^{2}\left(\sigma_{22}\right)_{\text {in }}+2 R_{21} R_{26}\left(\sigma_{16}\right)_{\text {in }}+2 R_{22} R_{26}\left(\sigma_{26}\right)_{\text {in }}+R_{26}^{2}\left(\sigma_{66}\right)_{\text {in }}
\end{aligned}
$$

The equations (A5) to (A8) express four of the $\sigma$-matrix elements at the exit, in terms of the $\sigma$ $\sigma$-matrix elements at the entrance. 
In order to prove part (a) of the APPENDIX 1 \{relations (A1) and (A2) \}, we make the following assumptions:

a) The incoming beam is achromatic, $\left\{\left(\sigma_{16}\right)_{\text {in }}=0,\left(\sigma_{26}\right)_{\text {in }}=0\right\}$ and the beam matches the dispersion of the transfer-line $\left(\eta_{\mathrm{x}}=0, \eta_{x}^{\prime}=0\right)$.

b) The R-matrix is a "pure" dipole of zero length, which provides the required dispersion and angular dispersion to the beam.

The R-matrix which describes such a dipole is given by the equation (A9) below.

$$
\begin{array}{ccc}
1 & 0 & R_{16} \\
0 & 1 & R_{26} \\
0 & 0 & 1
\end{array}
$$

The above two assumptions help separate the dispersive and non-dispersive part of the beam and is not loss of generality.

The use of the two assumptions above, reduce the equations (A5), (A6), (A7) and (A8) to the expressions below, which separate the dispersive part of the matrix element from the nondispersive.

$$
\begin{aligned}
& \left(\sigma_{11}\right)_{\text {out }}=\left(\sigma_{11}\right)_{\text {in }}+R_{16}^{2}\left(\sigma_{66}\right)_{\text {in }} \\
& \left(\sigma_{16}\right)_{\text {out }}=R_{16}\left(\sigma_{66}\right)_{\text {in }} \\
& \left(\sigma_{26}\right)_{\text {out }}=R_{26}\left(\sigma_{66}\right)_{\text {in }} \\
& \left(\sigma_{22}\right)_{\text {out }}=\left(\sigma_{22}\right)_{\text {in }}+R_{26}^{2}\left(\sigma_{66}\right)_{\text {in }} \\
& \left(\sigma_{12}\right)_{\text {out }}=\left(\sigma_{12}\right)_{\text {in }}+R_{16} R_{26}\left(\sigma_{66}\right)_{\text {in }}
\end{aligned}
$$

Equations (A10) to (A14) show the contribution of the dispersion and angular dispersion to the $\sigma$-matrix. In fact the dispersion $\left(\mathrm{R}_{16}, \mathrm{R}_{26}\right)$, introduced by the dipole of zero length is now the dispersion $\left(\eta_{\mathrm{x}}, \eta_{x}^{\prime}\right)$ of the transfer-line. This is because the beam was matched to the beam line in front of the dipole ${ }^{5}$.

Thus the equations (A10) to (A13) can be written as:

$$
\begin{aligned}
& \left(\sigma_{11}\right)_{\text {out }}=\left(\sigma_{11}\right)_{\text {in }}+\left(\eta_{x}^{2}\right)_{\text {in }}\left(\sigma_{66}\right)_{\text {in }} \\
& \left(\sigma_{16}\right)_{\text {out }}=\left(\eta_{x}\right)_{\text {in }}\left(\sigma_{66}\right)_{\text {in }} \\
& \left(\sigma_{26}\right)_{\text {out }}=\left(\eta_{x}^{\prime}\right)_{\text {in }}\left(\sigma_{66}\right)_{\text {in }} \\
& \left(\sigma_{22}\right)_{\text {out }}=\left(\sigma_{22}\right)_{\text {in }}+\left(\eta_{x}^{\prime 2}\right)_{\text {in }}\left(\sigma_{66}\right)_{\text {in }} \\
& \left(\sigma_{12}\right)_{\text {out }}=\left(\sigma_{12}\right)_{\text {in }}+\left(\eta_{x}\right)_{\text {in }}\left(\eta_{x}^{\prime}\right)_{\text {in }}\left(\sigma_{66}\right)_{\text {in }}
\end{aligned}
$$

5 The beam was achromatic and the dispersion of the line was zero in front of the dipole. 
The first terms of the RHS in equations (A15), (A18) and (A19) above correspond to the contribution of the non-dispersive part, $\left\{\right.$ namely $\left(\sigma_{11}\right)_{\text {in }},\left(\sigma_{22}\right)_{\text {in }}$, and $\left.\left(\sigma_{12}\right)_{\text {in }}\right\}$, to the matrix elements $\left(\sigma_{11}\right)_{\text {out }},\left(\sigma_{22}\right)_{\text {out }}$, and $\left(\sigma_{12}\right)_{\text {out }}$ and the second terms $\left\{\left(\eta_{x}^{2}\right)_{\text {in }}\left(\sigma_{66}\right)_{\text {in }},\left(\eta_{x}^{2}\right)_{\text {in }}\left(\sigma_{66}\right)_{\text {in }}\right.$ and $\left.\left(\eta_{x}\right)_{\text {in }}\left(\eta_{x}^{\prime}\right)_{\text {in }}\left(\sigma_{66}\right)_{\text {in }}\right\}$ corresponds to the contribution of the dispersion.

Note that the $\sigma$-matrix elements, expressed by the LHS of the equations (A15) to (A19) describe the same beam ellipsoid expressed by the $\sigma$-matrix elements on the LHS, because the dipole which introduced the dispersion had zero length. It is simply an alternative way of expressing the matrix elements.

In order to prove part (b) of the APPENDIX 1 we make use of equations (A15) to (A19) which separate the dispersive part of the $\sigma$-matrix from the non-dispersive part. Thus we make the following substitutions in the RHS of equation (A5).

$$
\begin{aligned}
& \left(\sigma_{11}\right)_{\mathrm{in}}=>\left(\sigma_{11}\right)_{\mathrm{in}}+\left(\eta_{x}^{2}\right)_{\mathrm{in}}\left(\sigma_{66}\right)_{\mathrm{in}} \\
& \left(\sigma_{22}\right)_{\mathrm{in}}=>\left(\sigma_{22}\right)_{\mathrm{in}}+\left(\eta_{x}^{2}\right)_{\mathrm{in}}\left(\sigma_{66}\right)_{\mathrm{in}} \\
& \left(\sigma_{16}\right)_{\mathrm{in}}=>\left(\eta_{x}\right)_{\mathrm{in}}\left(\sigma_{66}\right)_{\mathrm{in}} \\
& \left(\sigma_{26}\right)_{\mathrm{in}}=\left(\eta_{x}^{\prime}\right)_{\mathrm{in}}\left(\sigma_{66}\right)_{\mathrm{in}} \\
& \left(\sigma_{12}\right)_{\mathrm{in}}=>\left(\sigma_{12}\right)_{\mathrm{in}}+\left(\eta_{x}\right)_{\mathrm{in}}\left(\eta_{x}^{\prime}\right)_{\mathrm{in}}\left(\sigma_{66}\right)_{\mathrm{in}}
\end{aligned}
$$

Thus equation (A5) above can be written as:

$$
\begin{aligned}
\left(\sigma_{11}\right)_{\mathrm{out}}= & R_{11}^{2}\left(\sigma_{11}\right)_{\mathrm{in}}+2 R_{11} R_{12}\left(\sigma_{12}\right)_{\mathrm{in}}+R_{12}^{2}\left(\sigma_{22}\right)_{\mathrm{in}}\{\text { Non-Dispersive Contribution }\} \\
& +R_{11}^{2}\left(\eta_{x}^{2}\right)_{\mathrm{in}}\left(\sigma_{66}\right)_{\mathrm{in}}+2 R_{11} R_{12}\left(\eta_{x}\right)_{\mathrm{in}}\left(\eta_{x}^{\prime}\right)_{\mathrm{in}}\left(\sigma_{66}\right)_{\mathrm{in}}+R_{12}^{2}\left(\eta_{x}^{2}\right)_{\mathrm{in}}\left(\sigma_{66}\right)_{\mathrm{in}} \\
& +2 R_{11} R_{16}\left(\eta_{x}\right)_{\mathrm{in}}\left(\sigma_{66}\right)_{\mathrm{in}}+2 R_{12} R_{16}\left(\eta_{x}^{\prime}\right)_{\mathrm{in}}\left(\sigma_{66}\right)_{\mathrm{in}}+2 R_{16}^{2}\left(\sigma_{66}\right)_{\mathrm{in}}-R_{16}^{2}\left(\sigma_{66}\right)_{\mathrm{in}} \\
& =R_{11}^{2}\left(\sigma_{11}\right)_{\mathrm{in}}+2 R_{11} R_{12}\left(\sigma_{12}\right)_{\mathrm{in}}+R_{12}^{2}\left(\sigma_{22}\right)_{\mathrm{in}}\{\text { Non-Dispersive Contribution }\} \\
& +\left\{R_{11}\left(\eta_{x}\right)_{\mathrm{in}}+R_{12}\left(\eta_{x}^{\prime}\right)_{\mathrm{in}}+R_{16}\right\}\left\{R_{11}\left(\eta_{x}\right)_{\mathrm{in}}+R_{12}\left(\eta_{x}^{\prime}\right)_{\mathrm{in}}-R_{16}+2 R_{16}\left(\sigma_{66}\right)_{\mathrm{in}}\right\}\left(\sigma_{66}\right)_{\mathrm{in}}
\end{aligned}
$$

Substituting the expressions (A23) and (A24) into equation (A6)

$$
\begin{aligned}
\left(\sigma_{16}\right)_{\text {out }} & =R_{11}\left(\eta_{x}\right)_{\text {in }}\left(\sigma_{66}\right)_{\text {in }}+R_{12}\left(\eta_{x}^{\prime}\right)_{\text {in }}\left(\sigma_{66}\right)_{\text {in }}+R_{16}\left(\sigma_{66}\right)_{\text {in }} \\
& =\left\{R_{11}\left(\eta_{x}\right)_{\text {in }}+R_{12}\left(\eta_{x}^{\prime}\right)_{\text {in }}+R_{16}\right\}\left(\sigma_{66}\right)_{\text {in }}
\end{aligned}
$$

The condition of achromaticity at any section of the beam line requires:

$\left(\sigma_{16}\right)_{\text {out }}=0$ and $\left(\sigma_{26}\right)_{\text {out }}=0$

The above condition requires that the terms of the RHS in Equation (A27) sum up to zero.

$$
\left\{R_{11}\left(\eta_{x}\right)_{\text {in }}+R_{12}\left(\eta_{x}^{\prime}\right)_{\text {in }}+R_{16}\right\}=0
$$

The condition (A28) applied to (A26) yields:

$$
\left(\sigma_{11}\right)_{\text {out }}=R_{11}^{2}\left(\sigma_{11}\right)_{\text {in }}+2 R_{11} R_{12}\left(\sigma_{12}\right)_{\text {in }}+R_{12}^{2}\left(\sigma_{22}\right)_{\text {in }}\{\text { Non-Dispersive Contribution }\}
$$




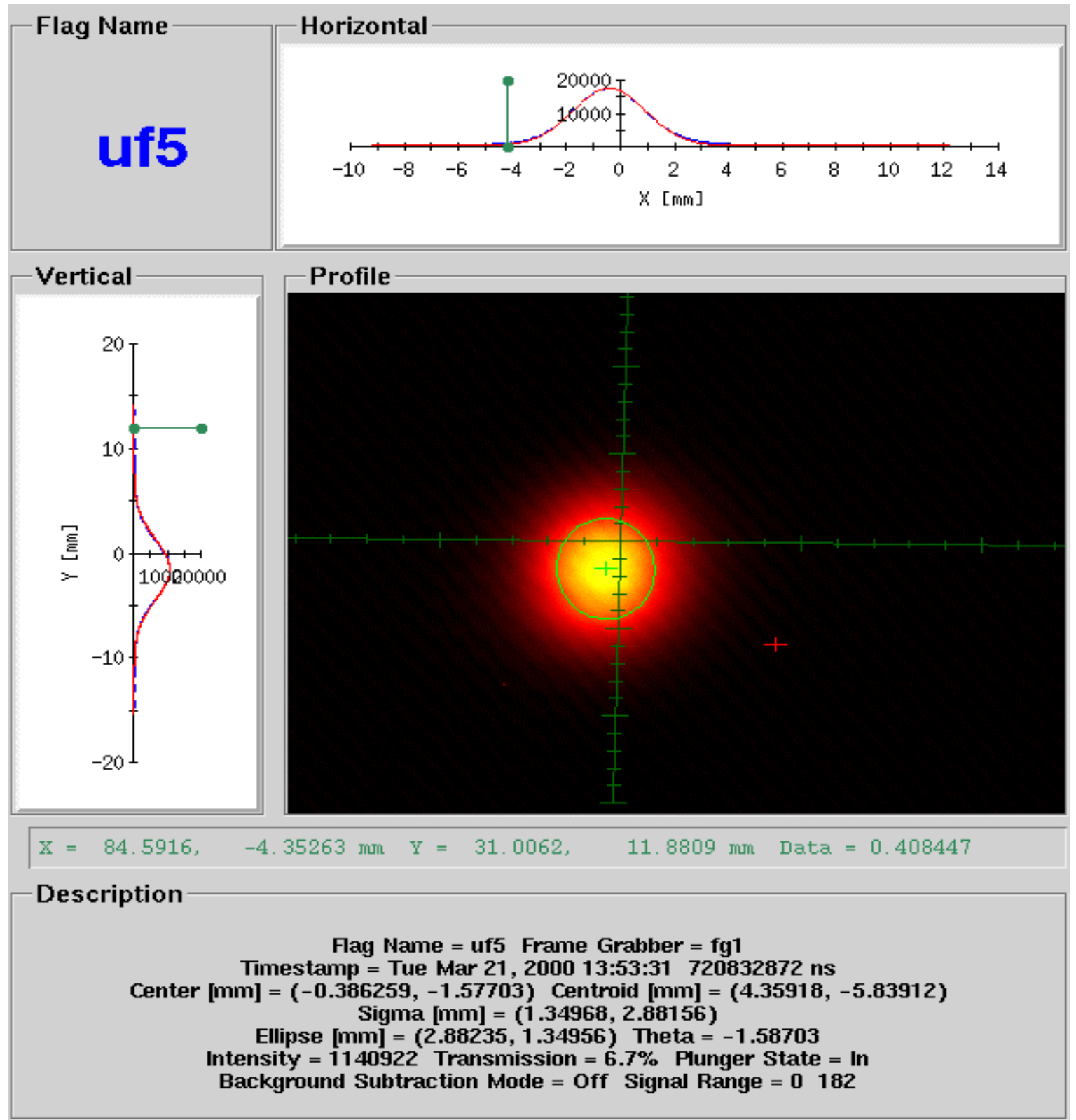

Figure $12 \mathrm{D}$ beam profiles and its projections (x,y beam profiles) obtain with the uf5 flag which is located at the end of the U-line. This window is displayed by the "ATR Flag Profile Monitor" application. In the "Description" section of the window the standard deviations "Sigma" of the x,y beam profiles are shown. 


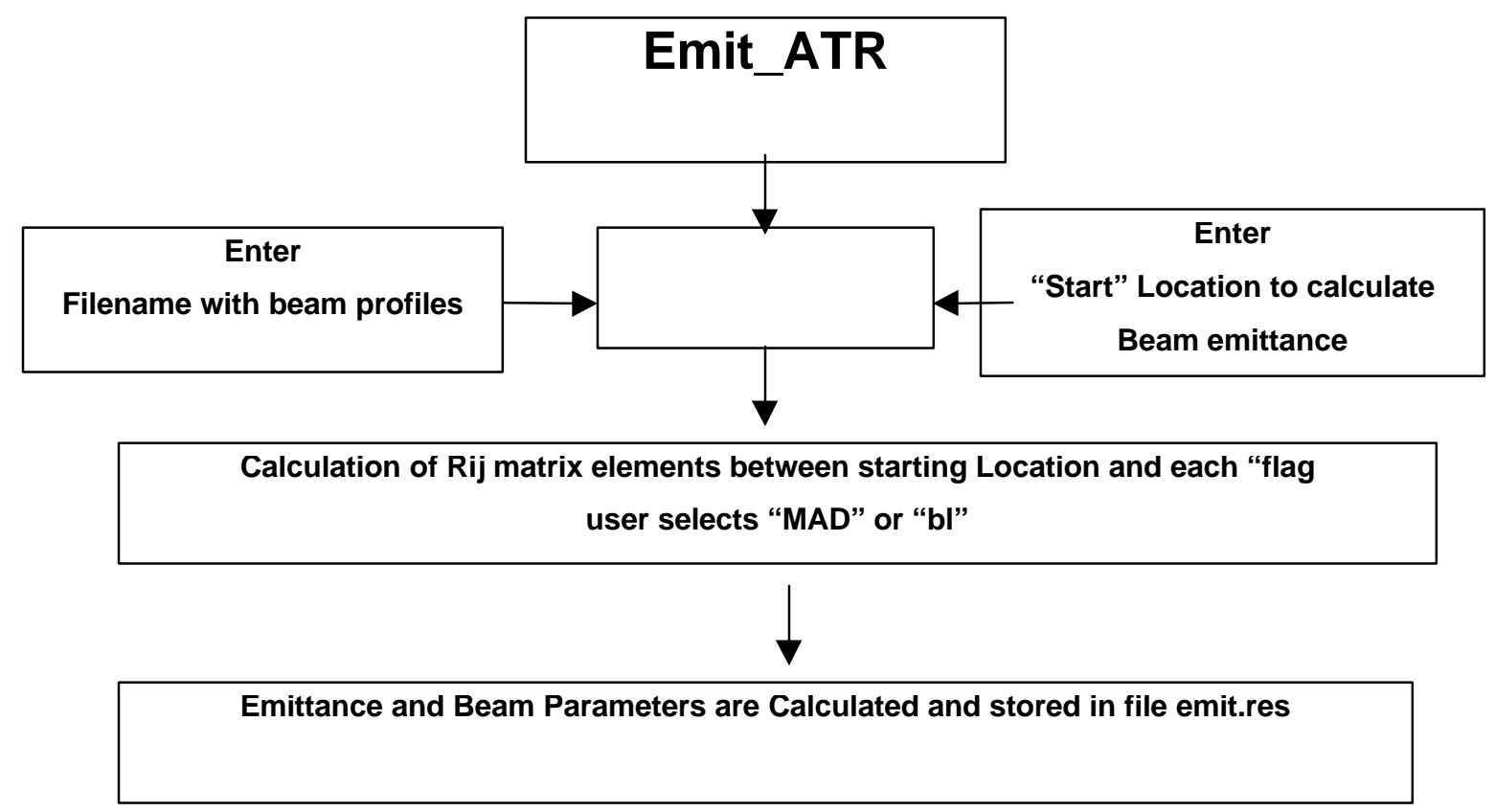

Figure 2. Flow-chart diagram of the program "emit-ATR" which calculates beam emittance and parameters from beam profiles measured with the "ATR Flaf Profile Monitor" application.

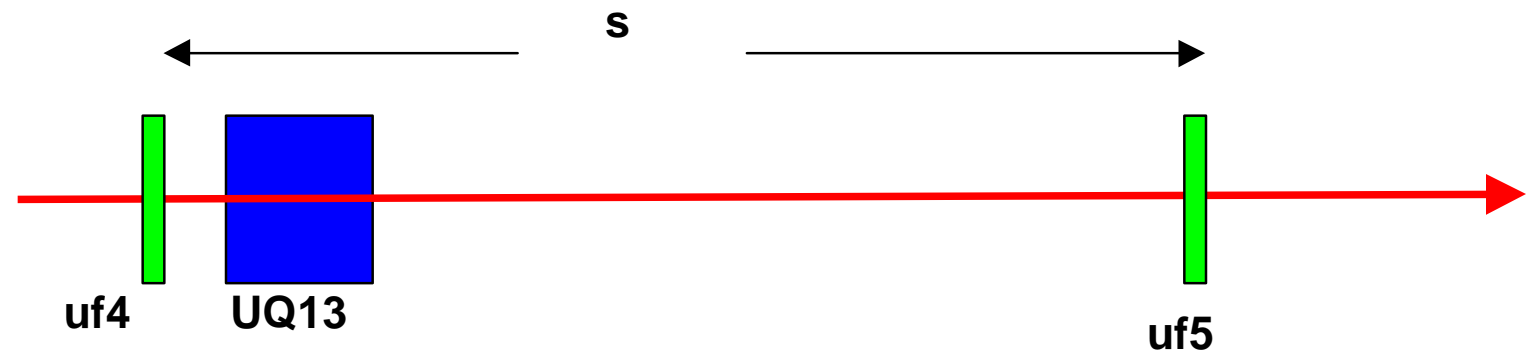

Figure 3. Schematic diagram showing the geometry of the flags and the quadrupole required to perform emittance measurement. The quadrupole is the rectangular box UQ13, and the narrow rectangular boxes are the flags uf 4 and uf5. The direction of the beam is shown by the arrow. The distance between the two flags is symbolized by s. 


\section{$\sigma_{x}{ }^{1 / 2}$ at uf5 vs KL of UQ13}

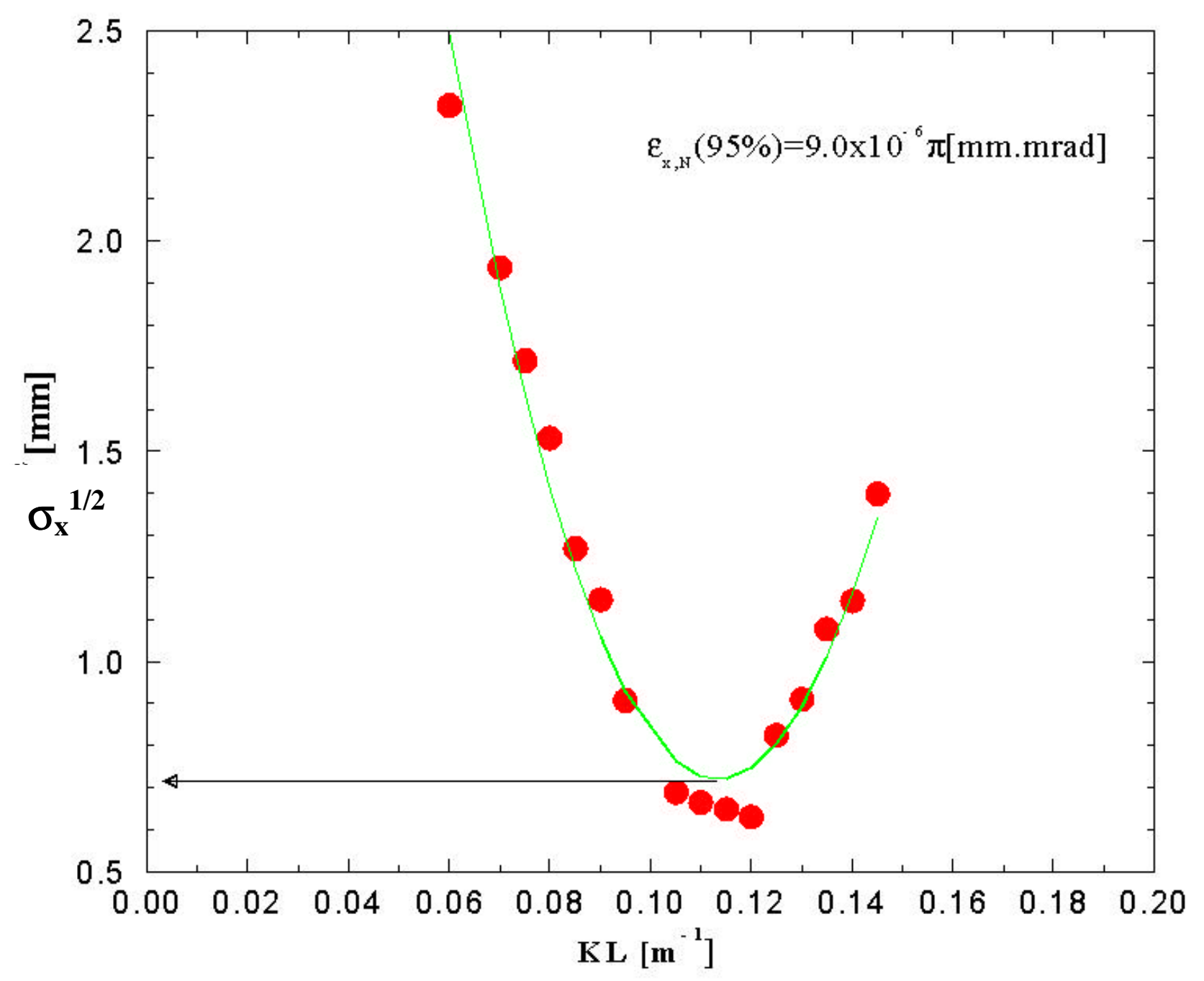

Figure 4 The standard deviation of the beam profile at the flag uf5 as a function of the quadrupole UQ13 strength. 